\title{
Gas of D-branes and Hagedorn density of BPS states
}

\section{Citation}

Vafa, Cumrun. 1996. "Gas of D-Branes and Hagedorn Density of BPS States." Nuclear Physics B 463 (2-3): 415-19. https://doi.org/10.1016/0550-3213(96)00025-9.

\section{Permanent link}

http://nrs.harvard.edu/urn-3:HUL.InstRepos:41385067

\section{Terms of Use}

This article was downloaded from Harvard University's DASH repository, and is made available under the terms and conditions applicable to Other Posted Material, as set forth at http:// nrs.harvard.edu/urn-3:HUL.InstRepos:dash.current.terms-of-use\#LAA

\section{Share Your Story}

The Harvard community has made this article openly available.

Please share how this access benefits you. Submit a story.

\section{Accessibility}


HUTP-95/A042

hep-th/9511088

\title{
Gas of D-Branes and Hagedorn Density of BPS States
}

\author{
Cumrun Vafa \\ Lyman Laboratory of Physics \\ Harvard University \\ Cambridge, MA 02138
}

\begin{abstract}
We test the prediction of a hagedorn density of BPS states which carry RR charge in type II compactifications. We find that in certain cases they correspond to the supersymmetric ground states for a gas of identical 0-branes.
\end{abstract}

Nov. 1995 
One of the key issues in understanding and testing string dualities is the question of BPS states. BPS states are stable even in strong coupling regimes in the theory and so if one finds BPS states in a given theory, it should also appear in any equivalent dual theory. This question becomes even more crucial when one notices that there is a hagedorn density of BPS states among perturbative states in many string theories [1]. An example of this phenomenon occurs for toroidal compactifications of type II strings. In such cases there are BPS multiplets corresponding to choosing oscillator ground states on one side and arbitrary oscillator state on the other.

In these (and many similar) cases the corresponding proposed duals do not exhibit the corresponding BPS states as elementary string states and one would like to understand how such a large degeneracy of states arises.

With the proposal of [2] it has become clear that the relevant BPS states not manifest in the perturbative type II spectrum should come from D-branes. This idea has been further developed in constructing bound states of D-branes in [3] leading to a surprising connection with the study of vacua for certain supersymmetric gauge theories. It was shown there that the spectrum of bound states of parallel 1-branes and strings is consistent with the prediction [4] of $S L(2, Z)$ duality of type IIB in ten dimensions. This development was continued in [5] where it was shown that even after toroidal compactification the story for parallel D-branes is consistent with the prediction of $U$-dualities [6]. Moreover, using the results of [7] for intersecting D-branes it was shown [8] that for the first oscillator level of BPS states the prediction of U-duality is consistent with the bound states of intersecting D-branes. In this note we will extend this result to all oscillator levels, and discuss some of its generalizations to type II-heterotic duality in 6 dimensions.

One of the cleanest cases for testing predictions of $U$-duality as far as BPS states is concerned is type II toroidal compactification down to 6 dimensions. This theory has $8 U(1)$ gauge fields coming from the NS-NS sector and 8 coming from the R-R sector. Moreover there is an element of order two in the $U$-duality group which exchanges these two sets of charges [9]. Let us consider the BPS states seen in the type II perturbation theory: The NS-NS $U(1)$ charges of BPS state is characterized by specifying a vector $\left(P_{L}, P_{R}\right)$ in the Narain lattice $\Gamma^{4,4}$ of the toroidal compactification. For each such charge the number of BPS states is given by taking the oscillator ground states on one side (say right) tensored with arbitrary oscillator state on the left with total oscillator number $N_{L}$. The level matching requires

$$
N_{L}=\frac{1}{2}\left(P_{R}^{2}-P_{L}^{2}\right)=\frac{1}{2} P^{2}
$$


Moreover the mass $m$ of the BPS state is given by $m^{2}=P_{R}^{2}$. Note that even if we know the existence of a BPS state only for a given $P=\left(P_{L}, P_{R}\right)$ the T-duality group $S O(20,4 ; \mathbf{Z})$ implies the existence of the same number of BPS states for any vector in $\Gamma^{4,4}$ which is in the same orbit as $P$.

Now we will be more specific: The $Z_{2}$ element of the $U$-duality group maps these momentum states to $D$-branes wrapped around the various cycles of $T^{4}$ [2]. Moreover the $P \cdot P$ gets mapped to the intersection number of $p$-branes. The case of parallel $p$-branes, where $P^{2}=0$ was studied in [3] (extended to the compact case in [5]) and was shown to agree with the prediction of $U$-duality. A particular case where $\frac{1}{2} P^{2}=1$ was considered in [8] by studying in the type IIA two 2-branes wrapped around 2-cycles of $T^{4}$, intersecting at one point in $T^{4}$. Note that by $T$-duality we could have equally well considered a 0 -cycle and a 4-cycle which again intersect at one point. This is more convenient for us and in fact we will generalize it by considering $m 0$-cycles and one 4 -cycle. This gives $\frac{1}{2} P^{2}=m$. Let us first review the result of $[8]$ for $m=1$. The expected degeneracy of BPS states in this case is $16^{3}$, a factor of 16 coming from right-moving ground state, another factor of 16 from the left-moving ground state, and a factor of 16 due to the degeneracy of the oscillators at $N_{L}=1$ over the left-moving ground state. To avoid the difficulties of bound states at threshold we first dualize type IIA to type IIB, as in [5] [8], where we end up having one 1-brane and one 5-brane and consider the extra direction of the branes wrapped an odd number of times around the extra dimension. The resulting supersymmetric quantum field theory now lives in different dimensions: From the 1-brane we get the reduction of $N=1 U(1) \mathrm{YM}$ from 10 to 2 dimensions [3], from the 5-brane, we get the reduction of $N=1 U(1)$ YM from 10 to 6 and from the joint configuration [7] we get a matter multiplet charged under the two $U(1)$ 's living on the common 2 dimensional space. In this case, given the triviality of the $U(1)$ dynamics, we are justified in ignoring the dynamics of the 6 dimensional theory and simply concentrating on the common 2-dimensional theory where the two theories interact which is the $d=2$ reduction of $N=2, d=4$ system with $U(1)_{r} \times U(1)_{c} / Z_{2}$ gauge group with adjoint matter $\Phi^{r}$ and $\Phi^{c}$ in each $U(1)$ and a hypermultiplet charged under $U(1)_{r}$. Moreover we end up with odd electric flux in the spatial direction for the two $U(1)$ 's due to the wrapping of the D-branes around the spatial direction [3] 8]. However one has to be careful to note that since we are dealing with compact space the bosonic component of $\Phi^{r}$ and $\Phi^{c}$ (or its dual) parameterize $T^{4}$. It was argued in [8] that the gauge system for $U(1)_{r}$ (in the sector with odd electric flux which cannot be screened by the matter which carries even charge) has a unique ground state. 
Quantizing the bosonic piece of $U(1)_{c}$ simply gives the space-time momenta. Quantizing the bosonic piece of $\Phi^{r}$ and $\Phi^{c}$ gives degeneracy one, because of the compactness of the variables. Quantization of the fermionic mode of $\Phi^{r}$ is the same as the cohomology of $T^{4}$ giving 16 states. The quantization of fermions in $U(1)_{c}$ and $\Phi^{c}$ (a total of 16 fermions) gives the extra $2^{8}=16^{2}$ degeneracy corresponding to left- and right-moving degeneracies of the ground state. Thus in total we get $16^{3}$ states as expected. We can do more, and actually check the spacetime quantum numbers of these states. They will also agree with the predictions of $U$-duality (when we recall that the fermions in the above are spacetime spinors).

Now let us consider the case where we have $m 0$-branes and $n 4$-branes. In the type IIB setup (where we dualize one of the directions and end up with $m$ 1-branes and $n$-branes) this would correspond to two quantum field theories, one living in 2 dimensions with gauge group $U(m)$ and one living in 6 dimensions with gauge group $U(n)$ and interacting on the common 2 dimensional space. Unlike the case with $n=1$ we cannot justify ignoring the non-abelian dynamics of the $U(n)$ theory in 6-dimensions and projecting down to 2 dimensions could miss some of the states which we would have gotten otherwise. However, if we take the case where we have $n=1$ but choose arbitrary $m$, then we are justified, as above in ignoring the dynamics of the 6 dimensional theory, as it is a free abelian theory, and simply concentrate on the common 2 dimensional theory where theory becomes interacting. Thus we will consider the case with $m$ 0-branes and one 4-brane. Then the system we need to consider is the $d=2$ reduction of $N=2, \mathrm{~d}=4$ with gauge symmetry $U(m) \times U(1)_{c}$ with an adjoint $\Phi_{1}$ of $U(m)$ and an adjoint $\Phi_{2}$ of $U(1)_{c}$, and a matter $\Lambda$ in fundamental of $U(m)$ 1. Note that we can think of eigenvalues of $\Phi_{1}$ as corresponding to the positions of the $m$ 0-branes on $T^{4}$ [3]. Note however that if the corresponding gauge theory has no mass gap, we still have to recall that the field space is compact because eigenvalues of $\Phi_{1}$ parameterize $T^{4}$ and we would then have in addition to quantize the collective coordinates for the positions of the 0-branes on $T^{4}$. In fact it is easy to see, using the argument of [8] that there is no mass gap. The background charges will cause the $U(1)$ of $U(m)$ to be broken, but the $S U(m)$ survives. Since we have matter in the fundamental of $S U(m)$ any charge at infinity will be screened leaving no mass gap. Another way of saying this is as follows: We can deform the $U(m) \times U(1)_{c}$ theory, by giving expectation value to the $\Phi_{1}$

1 Amusingly enough this is the same system that corresponds to the Calabi-Yau compactifications with the ground ring singularity of $c=1$ string at radius $R=m$ []]. 
field so that it breaks it to $U(1)^{m} \times U(1)_{c}$. The fundamental field $\Lambda$ now decompose to $m$ fields charged under each of the $U(1)^{m}$ 's. Moreover the background charge at infinity, which coupled to the diagonal $U(1)$ now couples to all these $U(1)$ 's and thus the argument of [8] would show that, apart from the choice of $\Phi_{1}$ we have $1^{m}=1$ vacuum. However we still have to quantize the collective coordinates which is, after recalling the connection between $\Phi_{1}$ and the points on $T^{4}$ and including its eight fermionic partners, the same as the ground states of the supersymmetric quantum mechanical system of $m 0$-branes on $T^{4}$. We also have to note that the 0-branes are identical (this also follows from the Weyl group of $U(m))$ and so the space we are quantizing will correspond to the cohomology of $\left(T^{4}\right)^{m} / S_{m}$ where $S_{m}$ is the permutation group on $m$ objects. This space is singular precisely where a number ( say $k$ ) of 0-branes coincide. The 'resolution' of this is dictated by the fact that the physics near these points is governed by an enhancement to $U(k)$. It is natural to believe this physical 'resolution' of the singularity is mirrored by the hyperkähler resolution of this space (in accord with $N=4$ supersymmetry in $d=2$ ) which agrees with the usual orbifold formula. In fact this was also the viewpoint adopted in [10] in connection with similar computations in testing the Olive-Montanen S-duality conjecture. Actually in [10] the cohomology of the (resolved) $\left(T^{4}\right)^{m} / S_{m}$ space was computed where it was noted that the degeneracy of the cohomology in this case is in one to one correspondence with the partition function of left-movers of the superstring! This is exactly what we need in order to obtain the expected degeneracy of the BPS states here (the extra $16^{2}$ states come from the quantization of the fermions in $U(1)_{c}$ and $\left.\Phi_{2}\right)$. Note that this connection tells us that the $k$-th quantum of left-moving oscillation for the superstring corresponds to a bound state of $k 0$-branes. Thus the hyperkähler resolution of this space in particular predicts that even though we have no mass gap in this quantum field theory, there are bound states of $k$ 0-branes at threshold (which in the orbifold language come from the twisted sectors of this theory).

We can also check the spacetime quantum numbers of the BPS states and we find that they agree with these bound states. This again follows by the fact that the fermions of the quantum mechanical system are spacetime spinors and the observation (see footnote on $\mathrm{p}$. 74 of [10]) that $\frac{1}{2}\left(F_{L}+F_{R}, F_{L}-F_{R}\right)$ quantum numbers of the cohomology of $\left(T^{4}\right)^{m} / S_{m}$ are the same as the light-cone helicities of the left oscillator states of type II superstring compactified on $T^{4}$ down to six dimensions (note that $F_{L}$ and $F_{R}$ correspond respectively to holomorphic and anti-holomorphic degrees of the cohomology shifted by half the complex dimension). If we consider the case $m=1$ we get the cohomology of $T^{4}$ which has sixteen 
elements with $6 \mathrm{~d}$ helicity assignments in agreement with $\frac{1}{2}\left(F_{L}+F_{R}, F_{L}-F_{R}\right)$. This is sufficient to guarantee that for $\left(T^{4}\right)^{m} / S_{m}$ we also get the correct helicity assignments for BPS states.

Note that if we changed the orientation of the 4-brane, the length squared of the charge vector will become $-m$; the analysis above continues to hold which implies we get the same degeneracy. However this now corresponds to the BPS states, whose oscillator states come from the right-movers. Given the picture of the left-oscillator states corresponding to the 'gas' of 0-branes in the presence of a 4-brane, and the right-oscillator states corresponding to the 'gas' of 0-branes in the presence of an anti-4-brane, it is tempting to think of strings as 'composites' of these BPS states. This is very suggestive indeed.

A similar story seems to repeat for the type IIA compactification on $K 3$ and its duality with heterotic string on $T^{4}$. In that case the heterotic string does contain BPS states among its elementary excitations, with the degeneracy growing as the left-mover oscillator modes of the bosonic strings. The corresponding analysis for the gauge degeneracy has not been done yet, but the following observation is very suggestive: If we consider $m 0$-branes and one 4-brane, the requisite degeneracy (apart from the obvious fermionic degeneracy) is the degeneracy of open bosonic strings at level $m+1$, which according to the result of [10] is the same 2 as the ground states of the supersymmetric gas of $m+1$ points on $K 3$. It remains to do the analysis of the gauge sector and verify the above picture.

I would like to thank A. Johansen, S. Kachru, A. Sen and E. Witten for valuable discussions. This research is supported in part by NSF grant PHY-92-18167.

2 This was the main motivation for my conjecture of the duality between type IIA on $K 3$ and heterotic strings on $T^{4}$. 


\section{References}

[1] A. Dabholkar and J. Harvey, Phys. Rev. Lett. 63 (1989) 478.

[2] J. Polchinski, preprint hep-th/9510017.

[3] E. Witten, preprint hep-th/9510135.

[4] J. Schwarz, preprints hep-th/9508143, hep-th/9509148, hep-th/9510086.

[5] A. Sen, preprint hep-th/9510229.

[6] C. Hull and P. Townsend, Nucl. Phys. B438 (1995) 109.

[7] M. Bershadsky, V. Sadov and C. Vafa, preprint hep-th/9510225.

[8] A. Sen, preprint hep-th/9511026.

[9] A. Sen and C. Vafa, preprint hep-th/9508064.

[10] C. Vafa and E. Witten, Nucl. Phys. B431 (1994) 3. 